\title{
Conceptualising the Sensory Classroom Teacher Questionnaire to strengthen inclusive education
}

\author{
Hannelie du Preez (PhD) \\ University of Pretoria, Faculty of Education
}

\begin{abstract}
Communal learning spaces provide an immense amount of sensory input. Exploring sensory ergonomics offer a framework to understand the behaviour and learning of children who experience sensory processing difficulties such as attention-deficit/hyperactivity disorder (ADHD). Teachers are responsible for creating communal learning spaces that mirror inclusive education principles. In response, a need to guide teachers in creating inclusive, conducive and supportive classrooms, a psychoeducational assessment instrument was conceptualised. The purpose of the Sensory Classroom Teacher Questionnaire (SCTQ) is to assess teachers' awareness of sensory stimuli that an environment elicit; and provide opportunities

for co-regulation, self-care and self-regulation to the child diagnosed with ADHD. A qualitative mode of inquiry with conceptual analysis as approach was utilised. Literature about sensory ergonomics, ADHD, sensory processing dysfunction and questionnaire design was purposively sampled. The latter was analysed to define sensory ergonomics as overarching construct, develop sub-scales and applicable test items. The SCTQ is self-administering, selfrating and multidimensional and consists of four preliminary sub-scales. The SCTQ was qualitatively defined and appraised by six cross-disciplinary specialists for content-validity evidence. By qualitatively exploring the SCTQ, as part of an integration strategy, the feedback improved, reduced and refined the items. The refined version has been acceded and will be piloted in the near future. Insight derived from this inquiry emphasises the importance of the 'conceptualisation phase' in instrument development. This phase "appears" obvious and self-explanatory, but if not explored intentionally can result in psychoeducational assessment instruments becoming ambiguous and ideologically disparaged.
\end{abstract}

Keywords: Attention-deficit/hyperactivity disorder (ADHD); Early childhood; Instrument validation; Sensory integration (SI); Learning environments; Sensory Classroom Teacher Questionnaire (SCTQ). 